Brit. J. industr. Med., 1951, 8, 271.

\title{
AN APPLIANCE FOR THE PREVENTION OF MERCURY POISONING
}

\author{
BY \\ FRANZ STOCKER \\ From the Medical Inspectorate of Factories, Ministry of Public Health, Vienna
}

Occupational mercury poisoning on a large scale is unlikely to be seen by many industrial medical officers in future. This was not always so, for in the latter part of the nineteenth century the condition was common in the metallurgical industries in which mercury was extracted from cinnabar, in mirror manufacture, in fire-gilding, in haircutting, in hat-making, and in the manufacture of thermometers, barometers, and glow-lamps. In these and also in the chemical industry quicksilver poisoning was often severe.

By the introduction of preventive measures, and as a result of the changes in manufacturing methods which were developed about that time, the number of cases was considerably reduced in the subsequent decades. In many undertakings mercury poisoning ceased to exist.

A classical example of such improvement is found in the mirror industry where harmless mercury compounds replaced the pure metal. Weyl (1908) has described this advance and states that in Fürth, then the centre of the German mirror industry, mercurialism had ceased to appear as early as 1907 , though, about a decade earlier, it had occurred frequently causing thousands of working hours to be lost.

Technical advances brought fundamental changes in the commercial and industrial structure of all countries, and at the same time a greater degree of safety in previously dangerous occupations. Nevertheless new possibilities of injury to health were arising.

As examples we may mention the introduction of mechanical drills in mining and tunnelling, the development of modern solvents in various industries and the use of $x$-rays for therapeutic and other purposes. All these have led to diseases which were partly or completely unknown to earlier generations. Doctors employed in industry must therefore be aware of the occupational risks arising from the methods used at any particular period, and must devise means of protecting those workers whose health might be affected, by planning necessary safeguards in the early stages of any new industrial process.

To the rapidly expanding electrical and machine industry, mercury is essential because of its peculiar physical properties. More attention should be paid to the risk of mercury poisoning in the filling, emptying, and repairing of apparatus such as vacuum pumps using mercury, mercury rectifiers or contact breakers, and also mercury vapour lamps and electro-medical apparatus.

Measures usually employed to prevent mercury poisoning include the provision of adequate ventilation and of smooth tables and floors without rifts or crannies, the prohibition of smoking or eating during working hours, an insistence on the washing of hands before meals, as well as a complete and thorough wash when work ceases, the care of the mouth, a medical supervision of various shifts of workers etc.

But it is not always possible to be sure that small quantities of mercury do not escape onto the tables and floor or collect in small rifts or crevices. This may easily occur when apparatus used in physics or in the electrical industry, such as thermometers and barometers, are being manipulated. If the ventilation is inadequate, which is often the case in small factories, particularly in winter, it means that the workmen are obliged to spend many months in an atmosphere containing mercury vapour.

This paper describes an appliance for collecting spilled mercury, used for many years by Siemens and Halske of Vienna, manufacturers of wireless transmitters, electrical machines, measuring equipment, and similar apparatus. In its testing departments specially trained technicians examine the articles manufactured before delivery. Fig. 1 


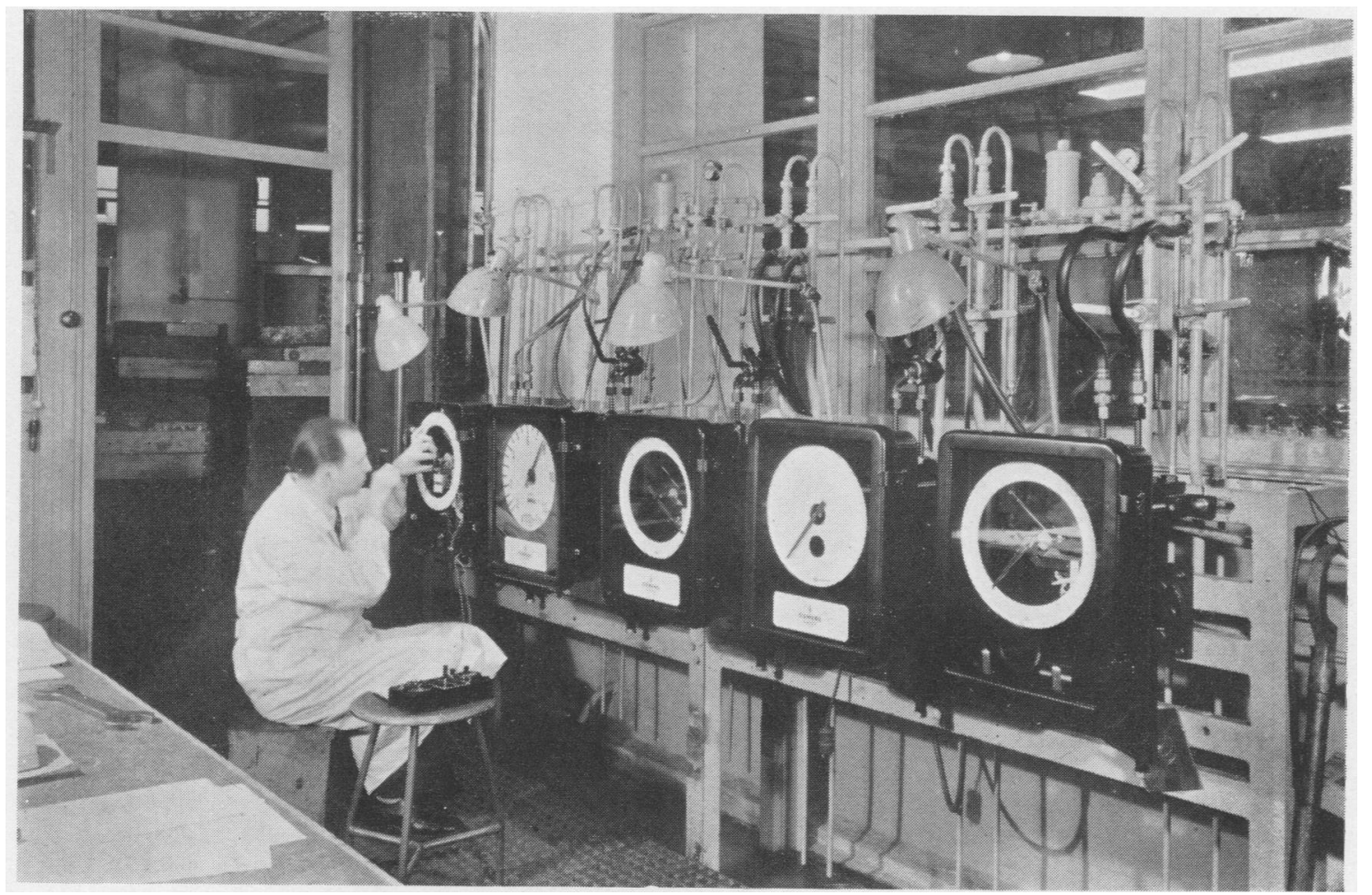

FIG. 1

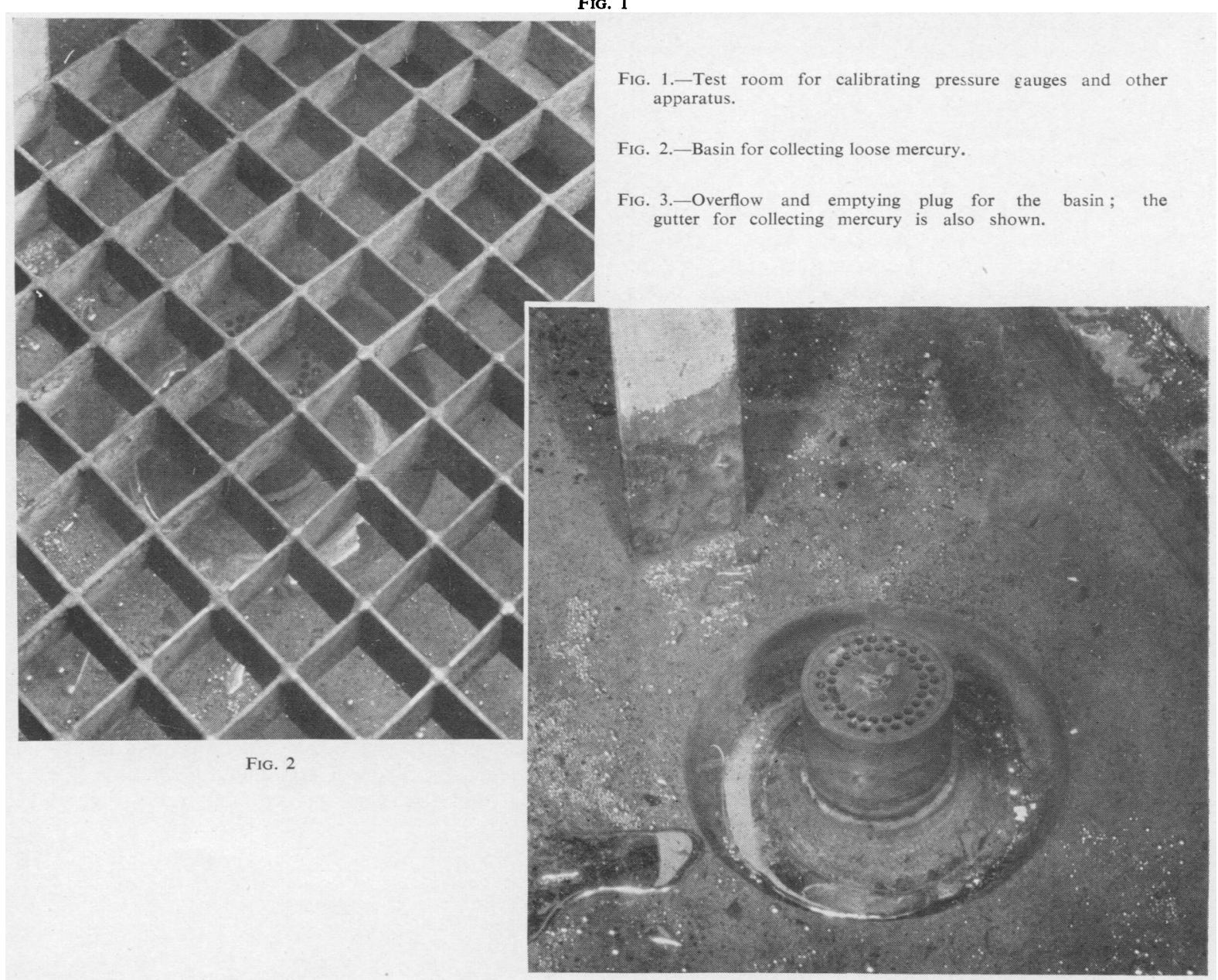



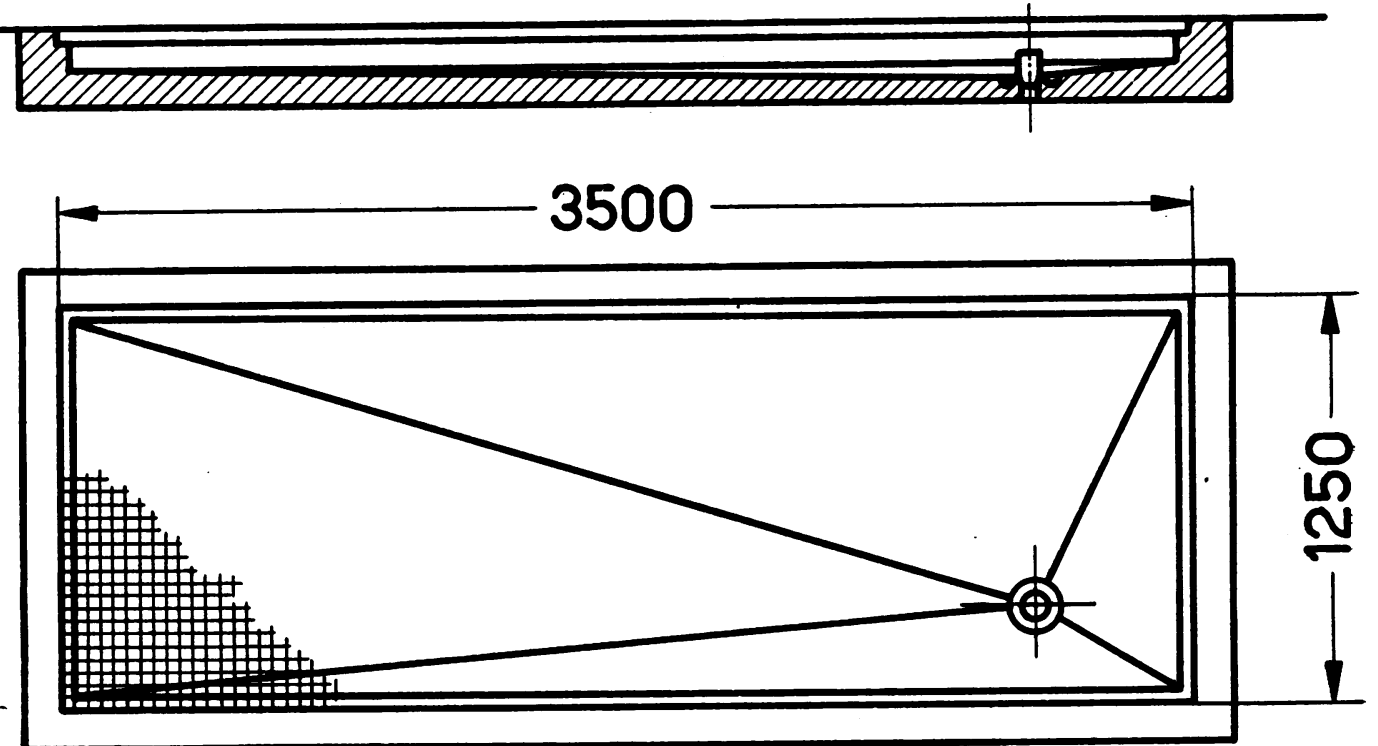

FIG. 4.-Plan and section of the collecting basin; dimensions are given in $\mathrm{cm}$.

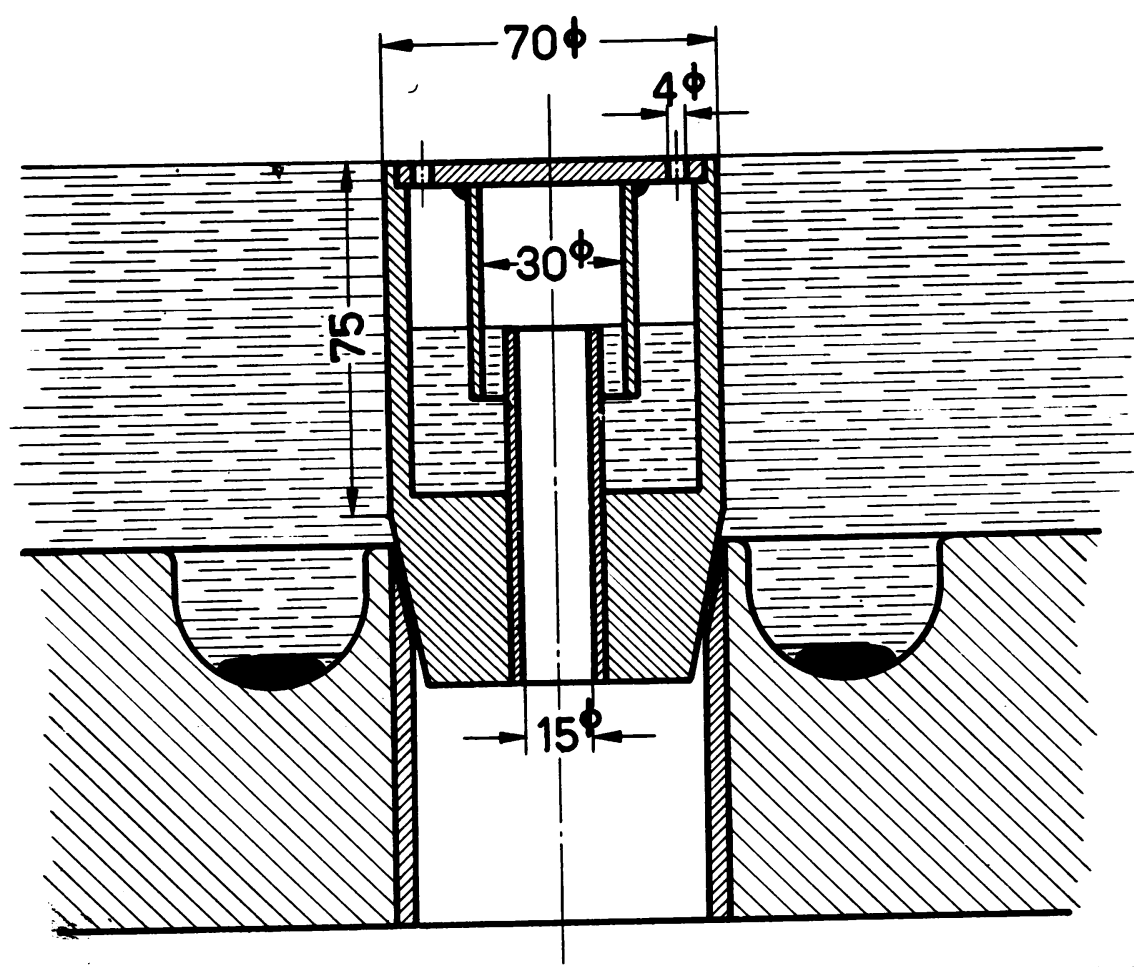

Fig. 5.-Cross-section through the overflow and emptying plug of the basin; the gutter for collecting mercury is also illustrated. 
shows one of these departments in which experts calibrate pressure gauges and apparatus used for quantity measurements. The apparatus contains up to $5 \mathrm{~kg}$. of mercury which is put into it and withdrawn by the operative. Even if the greatest possible care is taken, it is very difficult to prevent some drops of mercury from falling on the floor. A very simple safety device has made it possible to control this hazard. It consists of a large basin full of water which is let into the floor beneath the working point (Fig. 1) covered by an iron grill (Fig. 2). If, as often happens, some mercury is scattered while an apparatus under test is being filled or emptied, then the metal will fall through the grill into the basin which contains water. Fig. 3 shows the quantity of mercury which has fallen into the basin during a fairly short working period. The water is "syphoned off" through the plug in the centre of the gutter in which the mercury collects. The mercury is then easily removed and purified for further use.
Fuller details of the design and dimensions of the collecting trough are given in Figs. 4 and 5.

This apparatus has proved to be successful. The employee is completely protected, and is spared the anxiety and dangers of an insidious hazard. Because of its low cost the installation can easily be used by small firms, and it also represents an economy as mercury is recovered. The costs of medical examination by the authorities charged with industrial welfare are also saved. Such costs are normally borne by the employer, but are avoidable and can be considerably reduced when the precautions described are taken. The method described has already proved to be of value in preventing mercury poisoning, and for this reason we feel justified in making this appliance more widely known in industrial circles.

\section{REFERENCE}

Weyl, T. (1908). Handbuch der Arbeiterkrankheiten, Jena. 